The influence of the representative volume element (RVE) size on the homogenized response of cured fiber composites

This article has been downloaded from IOPscience. Please scroll down to see the full text article. 2012 Modelling Simul. Mater. Sci. Eng. 20075007

(http://iopscience.iop.org/0965-0393/20/7/075007)

View the table of contents for this issue, or go to the journal homepage for more

Download details:

IP Address: 141.211.173.82

The article was downloaded on 25/06/2013 at 20:33

Please note that terms and conditions apply. 


\title{
The influence of the representative volume element (RVE) size on the homogenized response of cured fiber composites
}

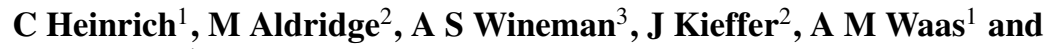 \\ K Shahwan ${ }^{4}$ \\ ${ }^{1}$ Department of Aerospace Engineering, University of Michigan, Ann Arbor, MI 48109, USA \\ ${ }^{2}$ Department of Materials Science and Engineering,University of Michigan, Ann Arbor, MI \\ 48109, USA \\ ${ }^{3}$ Department of Mechanical Engineering,University of Michigan, Ann Arbor, MI 48109, USA \\ ${ }^{4}$ Chairman - Board of Directors, Automotive Composites Consortium-ACC, and Chairman - \\ Predictive Technology Development and Composites Crash Energy Management Group \\ (ACC100), USCAR, Southfield, MI, 48075, USA \\ ${ }^{5}$ Chrysler Technology Center, Chrysler Group LLC, Auburn Hills, MI 48326-2757, USA \\ E-mail: dcw@umich.edu
}

Received 12 October 2011, in final form 25 July 2012

Published 14 September 2012

Online at stacks.iop.org/MSMSE/20/075007

\begin{abstract}
The influence of the representative volume element (RVE) size (in terms of fiber packing and number of fibers for a given fiber-volume fraction) on the residual stresses created during the curing process of a continuous fiber-reinforced polymer matrix tow is investigated with the ultimate goal of finding a minimum unit cell size that can be used later for a homogenization procedure to calculate the response of woven fiber textile composites and in particular, fiber tows. A novel network curing model for the solidification of epoxy is used to model the curing process. The model takes into account heat conduction, cure kinetics and the creation of networks in a continuously shape changing body. The model is applied to the curing of a fiber/matrix RVE. The results for the minimum size of the RVE, obtained on the basis of the curing problem, are compared with a similar RVE, modeled as an elastic-plastic solid subjected to external loads, in order to compare the minimum RVE sizes obtained on the basis of different boundary value problem solutions.
\end{abstract}

(Some figures may appear in colour only in the online journal)

\section{Introduction}

The increasing demand for lightweight structures in aerospace, automotive and other applications drives the need for sophisticated and optimized composite materials. At the same time a high-fidelity computational framework is required to analyze, evaluate and predict the ensuing deformation response when such composite materials are used in service. A typical 
representative of such a composite material is a carbon fiber textile composite with an epoxy matrix material. In order to optimize the material for its intended use, the structural part to be manufactured can be optimized with respect to geometrical properties, fiber architecture, thickness and lay-up. The manufacturing process can play an important role, as this can have a significant impact on the subsequent part performance during the application of service loads, because the manufacturing process can introduce self-equilibrating internal stresses within the material. If the process is modeled and understood properly, then the desired internal stress state can be 'designed' so that subsequent part performance is optimized. Such an approach has precedent in the manufacturing process of auto-glass, where compressive surface stresses are 'built-in' by tailoring the manner by which the cooling process is controlled, as shown in the pioneering work by Lee et al [1]. At the same time, it is shown that different cooling rate histories can produce undesired surface tensile stresses, which if present, can accelerate the propagation of surface flaws such as cracks, when tensile service loads are experienced.

Manufacturing-induced effects in composites have usually been studied in the context of cure induced stresses and potential failure during cure. Rabearison et al [2] give an example of how gradients during cure develop in a thick carbon epoxy tube and subsequently cause defects such as cracks. Rabearison et al then developed a numerical model for curing of an epoxy. They calculate the curing of the epoxy in a glass beaker and conclude that stresses large enough to cause cracks are developed during the curing process. In a similar fashion Corden et al [3] have developed a curing model to predict the residual stresses in thick resin transfer molded laminated cylinders. They identified chemical shrinkage due to cure as a major contributing mechanism for stress build-up as they cured all parts at room temperature. During the experimental validation of the results with different laminate layouts it was noted that interlaminar cracking can occur due to differential shrinkage. Plepys and Farris [4] noted the creation of cracks during isothermal cure of three-dimensionally constrained epoxy resin in a glass cylinder.

The generation of stresses during cure can lead to unintended geometric alterations of a component. For example L-shape parts may observe a 'spring-in' upon removal from the mold. Here the two sides of the L-shaped part bend inward giving a smaller angle then the anticipated $90^{\circ}$ [5-8]. Equivalently when a composite part is cured on a flat tool, bending or warpage can be observed due to thermal gradients and residual stresses that are built-up during cure [9-11].

In this study, the specific composite of interest is a $2 \mathrm{D}$ triaxially braided polymer matrix textile (2DTBC). Here one tow of carbon fibers runs in the axial direction and two bias tows are woven around it. A typical representative architecture is given in figure 1, which shows the hierarchical scaling that is present, from a macro-scale structure to a fiber tow unit cell that has several fibers impregnated with matrix material. It has been noted previously that the overall mechanical response of the cured composite does not reflect the one that could be expected from modeling the individual constituents [12], unless effects due to the curing process are accounted for. The curing process can cause structural changes to the matrix properties when the matrix is cured in the presence of fibers. These changes can also include residual stresses that can be built-in due to the complex interaction between fibers tows, matrix and non-uniform thermal fields during the curing process. In these studies [12] a representative unit cell has been considered at the tow level, and its effective properties have been inferred on the basis of different testing protocols carried out at the composite level. In this study, the focus is on the state of a cured matrix and the fibers within a fiber tow. It has been noted in previous related studies that the macroscopic response of a fiber tow is dependent on the shape and packing of the fibers $[13,14]$. Therefore, the objective of this paper is to understand the state of stress evolution within a fiber tow representative volume element (RVE) due to curing as a function 


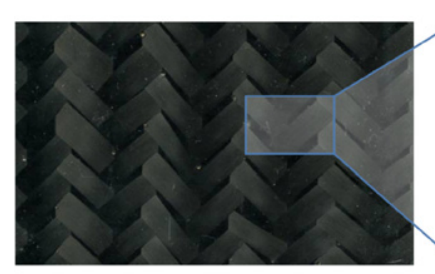

$45^{\circ} 2 \mathrm{D}$ triaxially braided composite

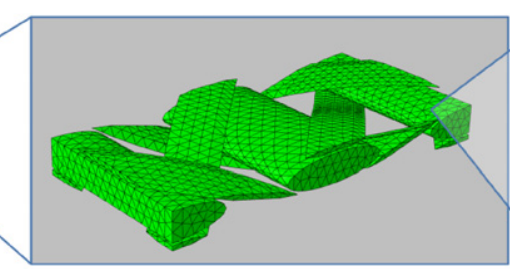

FE model of tow level unit cell

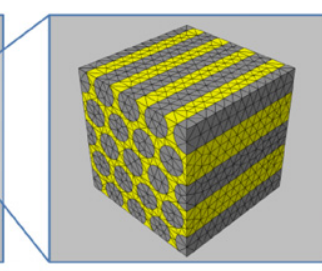

FE model of fiber level unit cell at each

Figure 1. Multiscale model: from braided composite (left) to tow level unit cell (middle) to fiber/matrix level unit cell of a tow (right).

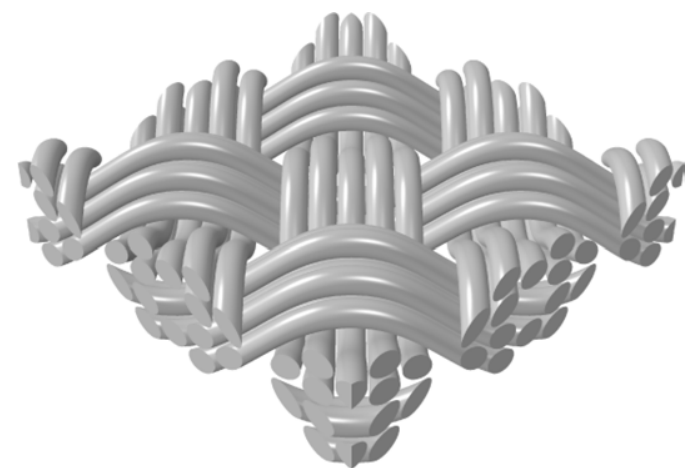

Figure 2. Plain weave composite modeled with discrete fibers.

of fiber packing. The goal is to find the minimum amount of fibers necessary to represent such a tow, such that the overall magnitude of stresses generated does not change significantly with an increase in RVE size. The 'converged' RVE size can then be used confidently during subsequent multiscale simulations. A related question that arises is whether the minimum size of RVE that is subsequently determined on the basis of solving an inelastic response boundary value problem (BVP) is similar to that obtained from solving the curing problem that is addressed in this paper.

As computational tools available to engineers become more powerful it also becomes possible to model fiber bundles and layers by discrete fibers. Figure 2 shows an example of a plain weave composite where the fiber tows are represented by seven discrete fibers. The results derived in this paper may also serve as a guideline of how many discrete fibers per tow are necessary to obtain a converged result.

The paper is organized as follows: section 2 discusses various homogenization methods and how randomness can be quantified. Then a material model is introduced to describe and quantify the phase change of an epoxy material. Section 4 shows how to find material properties for the proposed curing model from Raman and Brillouin light scattering data obtained from experiments that studied the epoxy-curing process. In section 5 details of the finite element based numerical study are presented. The paper concludes with a discussion of the results obtained and a summary of the key findings.

\section{Homogenization and micromechanics}

There are numerous techniques that can be used to obtain homogenized elastic and inelastic constitutive models of fiber-reinforced composite materials. A good summary of various 
homogenization methods for the elastic response is given in [15]. These techniques include rule of mixtures, concentric cylinder assemblage model [16], method of cells [17], the generalized method of cells [18-20] and the Mori-Tanka model [15]. These methods idealize the microstructure of the material, and most of them assume a regular pattern of fiber placement within the matrix material.

In reality the actual microstructure of the material is random in nature due to the manufacturing process. For example, the fiber packing within a fiber tow in a textile composite will neither be entirely random nor entirely regular but instead fiber clusters and matrix pockets can be found [21]. When including non-linear effects, different microstructures can have a profound impact on the overall result. Lissenden et al investigated the inelastic flow of fiberreinforced metals having different microstructures [22]. Specifically rectangular, hexagonal and square diagonal fiber packing arrays were investigated. It was found that the influence of microstructure is largest when fiber-matrix debonding is not an issue. Additionally, the effect of the microstructure increases with increasing fiber-volume fraction. A similar study was conducted by Brockenbrough et al [13]. In addition to the effect of the fiber shape, they also studied microstructures with a random fiber distribution. It was observed that there is a significant difference between the different packings investigated. In these studies, it is noted that the boundary conditions were chosen in such a way that straight boundary faces of the cross-sections considered remained straight during deformation. Recent studies of the micromechanical response of composites take a random distribution of fibers into account. These studies typically make use of the finite element method. González and Llorca [23] investigated the mechanical behavior of unidirectional fiber-reinforced polymers under transverse compression. Interface decohesion between fiber and matrix, and matrix plastic deformation were included as damage modes. Swaminathan et al [24] and Swaminathan and Ghosh [25] investigated the size of a statistically equivalent volume elements for a unidirectional composite with and without interfacial debonding. For their choice of material parameters they concluded that 50 fibers should be used in a RVE to determine the elastic properties correctly. When interfacial debonding is included, they observed that the smallest statistically meaningful RVE depends on the macroscopically applied strain and increases with increasing strain. The need for a minimum representative volume size when quantifying effective material properties of material exhibiting random patterns has also been noted by Rakow and Waas [26]. They have shown that the shear modulus of aluminum foam approaches the bulk representative value when a region larger than 18 times the characteristic cell diameter is used. Other studies regarding the effect of randomness in the distribution of particles and fibers in a RVE include [27-29].

A question that arises when taking into account randomly distributed fibers is how to quantify that randomness. Buryachenko et al [30] used a digital image processing technique to measure the coordinates of fibers. Then they estimated the statistical parameters and used radial distribution functions describing the random nature of the composite. Ghosh et al [31] noted that the radial distribution function and second order intensity functions are less suited to characterize the microstructure at higher volume fractions. Instead they employed Voronoi cells to model a multiphase material. Such a Voronoi tessellation with nine distinct fibers is shown in figure 3 on the left. The actual RVE is shaded in gray. To calculate the size of the Voronoi polygons within the periodic unit cell, an image of the fiber positions have to be created around the unit cell. These shifted copies are lying in the white region of figure 3 . The resulting FE model is shown on the right. The variation in the area of the Voronoi cells can be used to describe the deviation from regular packing. 

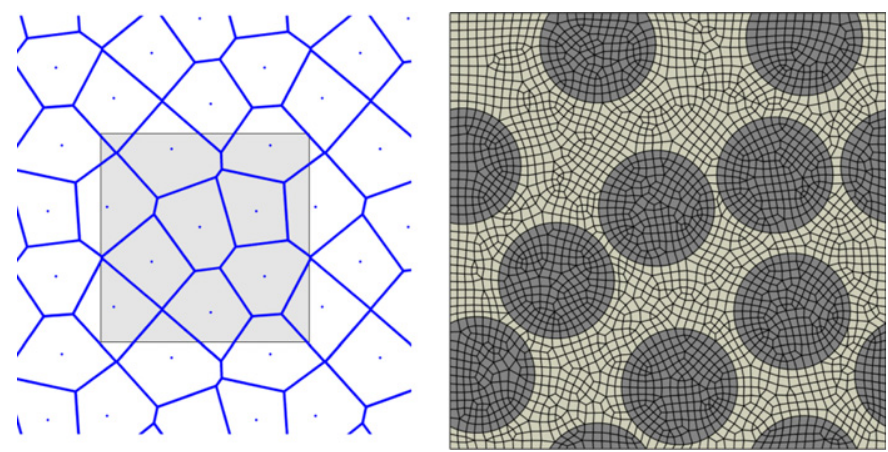

Figure 3. Voronoi cells and corresponding finite element model.

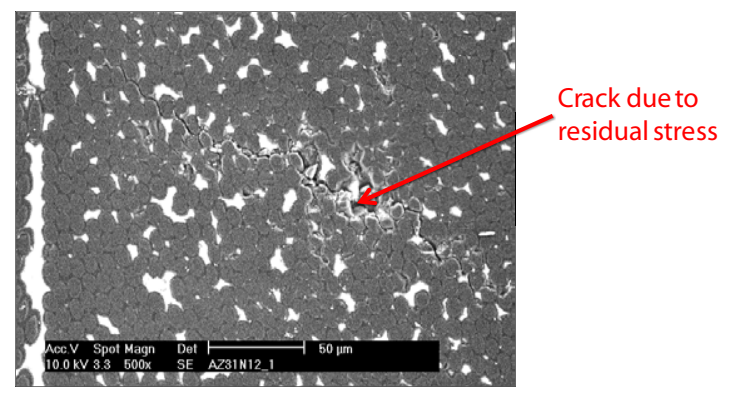

Figure 4. Cracks due to residual stresses.

\section{Material model}

The material under consideration is a 2D triaxially braided composite (2DTBC), but the methods discussed in this paper are equally applicable to laminated composites. Carbon fibers in a polymer (epoxy) matrix is considered. It has been found that the effective properties of a Hetron ${ }^{\circledR}$ matrix material in a composite is different from its bulk properties [12]. This is attributed to damage that can occur during the curing process. A SEM image within the tow of such a cured braided composite can be seen in figure 4. The material developed cracking during the curing step. No external loads have been applied yet. Such behavior is believed to stem from thermal mismatch of the different composite constituents, and shrinkage of the epoxy as it undergoes a phase transition from a liquid to a solid.

In the following, the entire manufacturing and curing process has to be considered. During the manufacturing process of the $2 \mathrm{DTBC}$, fiber mats are laid out in a mold. The epoxy resin is mixed with hardener and distributed in the mold, which is then closed and compressed with a specific prescribed temperature at the boundary. The exothermic nature of the curing reaction combined with a low thermal conductivity of the epoxy can lead to inhomogeneities in temperature which is also linked to the rate of reaction and amount of conversion and therefore to the different mechanical properties and stress build-up during cure. The typical curing problem can be broken down into three different phases: thermal evolution, chemical evolution and stress evolution. Only the generation of stress during cure is addressed in this paper. The incorporation of failure of the polymer during cure is described elsewhere [32, 33]. Furthermore, non-uniformity in the temperature field within the composite will be considered in order to account for the network formation that occurs during curing. 


\subsection{Heat conduction and curing}

The evolution of temperature and degree of cure are intimately linked. The curing of an epoxy is an exothermic chemical reaction (see for example studies regarding differential scanning calorimetry (DSC) of epoxy [34-36]). The degree of cure is often measured by placing a small sample in a digital scanning calorimeter, maintaining the sample at a constant temperature and measuring the heat that is generated during cure. Denote the heat generated up to time $t$ by $H(t)$ and the degree of cure by $\phi(t)$, both at time $t$. The degree of cure is often defined [37] by

$$
\phi(t)=\frac{H(t)}{H_{r}}
$$

where $H_{r}$ is the total heat that is generated. Thus, $\phi(t)$ monotonically increases from $\phi=0$, at the uncured state, to $\phi=1$, at the fully cured state. Note that the rate of heat generation per unit mass is

$$
r=\frac{\mathrm{d}\left(H_{r} \phi\right)}{\mathrm{d} t}
$$

The curing process is modeled by a kinetics equation of the form

$$
\frac{\mathrm{d} \phi}{\mathrm{d} t}=f(T, \phi)
$$

where $T$ is the temperature and $f(T, \phi) \geqslant 0$. Kamal [37] has suggested that $f(T, \phi)$ be given by

$$
\begin{aligned}
& f(T, \phi)=\left(k_{1}(T)+k_{2}(T) \phi^{m}\right)(1-\phi)^{n}, \\
& k_{1}(T)=A_{1} \exp \left(-\frac{\Delta E_{1}}{T R}\right), \\
& k_{2}(T)=A_{2} \exp \left(-\frac{\Delta E_{2}}{T R}\right),
\end{aligned}
$$

where $m$ and $n$ are constants, $R$ is the gas constant, $A_{1}, A_{2}$ are frequency-like constants and $\Delta E_{1}, \Delta E_{2}$ are activation energies. Choices for $m, n, k_{1}(T)$ and $k_{2}(T)$ for various epoxy-curing agent systems have appeared in the literature. The values for EPON 862 are summarized in table 1. $k_{1}(T)$ and $k_{2}(T)$ are fitting constants that are found in the Arrhenius equation, which is used to describe a wide variety of chemical reactions. The exponential portion is related to the probability that a reaction might take place during a collision of atoms. The activation energy describes that energy barrier that needs to be overcome for a reaction to take place. With increasing temperature the kinetic energy of individual atoms increases and it becomes easier to overcome the energetic barrier. In applications, equation (3) is used to describe the curing process even when the temperature varies with time [36].

In a curing structural composite, there is both local heat generation due to curing and heat conduction due to the presence of an external surface or along fibers. This process is governed by the local form of the first law of thermodynamics [38, 39]:

$$
\rho \frac{\mathrm{d} e}{\mathrm{~d} t}=-\frac{\partial q_{i}}{\partial x_{i}}+\rho r+\sigma_{i j} \frac{\mathrm{d}}{\mathrm{d} t} \varepsilon_{i j},
$$

where $e$ is the internal energy per unit mass, $\rho$ is the current mass density, $q_{i}$ are the components of the heat flux vector, $r$ is the rate of heat supply per unit mass, $\sigma_{i j}$ are the stress components and $\varepsilon_{i j}$ are the components of the infinitesimal strain tensor.

The internal energy is assumed to be proportional to temperature, $e=c T$, where $c$ is the specific heat capacity. The heat flux vector is related to the temperature gradient by the Fourier law of heat conduction,

$$
q_{i}=-\kappa \frac{\partial T}{\partial x_{i}}
$$


Table 1. Material properties of the curing epoxy obtained from experiments.

\begin{tabular}{ll}
\hline Frequency constant $A_{1}$ & $3.62 \times 10^{11} 1 / \mathrm{s}$ \\
Frequency constant $A_{2}$ & 0 \\
Activation energy $\Delta E_{1}$ & $88.5 \mathrm{~kJ} \mathrm{~mol}^{-1}$ \\
Activation energy $\Delta E_{2}$ & 0 \\
Heat of reaction $H_{r}$ & $230 \mathrm{~kJ} \mathrm{~kg}^{-1}$ \\
Curing exponents $m, n$ & 1.0 \\
Initial bulk modulus $K_{\mathrm{S}}$ & $5.0 \mathrm{GPa}$ \\
Final bulk modulus $K_{\mathrm{f}}$ & $6.6 \mathrm{GPa}$ \\
Initial shear modulus $\mu_{\mathrm{s}}$ & $0.01 \mathrm{GPa}$ \\
Final shear modulus $\mu_{\mathrm{f}}$ & $1.8 \mathrm{GPa}$ \\
Cure shrinkage $\varepsilon_{\mathrm{c}}$ & $2 \%$ \\
\hline
\end{tabular}

where $\kappa$ is the thermal conductivity. It is possible that the thermal conductivity depends on the degree of cure and the temperature, i.e. $\kappa=\kappa(T, \phi)$. Equation (7), with (2) and (8), becomes

$$
\rho c \frac{\partial T}{\partial t}=\frac{\partial}{\partial x_{i}}\left(\kappa(\phi, T) \frac{\partial T}{\partial x_{i}}\right)+\rho H_{r} \frac{\partial \phi}{\partial t}+\sigma_{i j} \frac{\mathrm{d}}{\mathrm{d} t} \varepsilon_{i j} .
$$

For the applications considered here, the rate of mechanical work, $\sigma_{i j} \mathrm{~d} \varepsilon_{i j} / \mathrm{d} t$, is assumed to be negligible. These applications may include a wide range of situations involving the cure of epoxy, ranging from a simple lab experiment to the manufacturing of composite structures, consisting of epoxy and fibers. With the assumption of negligible mechanical work, equation (9) becomes

$$
\rho c \frac{\partial T}{\partial t}=\frac{\partial}{\partial x_{i}}\left(K(\phi, T) \frac{\partial T}{\partial x_{i}}\right)+\rho H_{r} \frac{\partial \phi}{\partial t} .
$$

Equations (3), (4) and (10) form a system of coupled non-linear partial differential equations for the spatial distribution and time variation of temperature, $T(\underline{x}, t)$, and degree of cure, $\phi(\underline{x}, t)$.

\subsection{Stress development}

To investigate stress development, a network model of a polymer will be adopted. The networks interpenetrate each other, i.e. they occupy the same volume. On the other hand, the assumption is made that they do not interact with each other.

A second assumption is related to the interaction of the different physical effects mentioned earlier: heat conduction, chemical change and mechanical response. Curing of epoxy resins is often carried out under pressure. This has influence on the rate of cure and can be taken into account. Ramos et al [40] measured and modeled the cure kinetics and shrinkage for a DGEBA/ MCDEA epoxy system. The reaction rate was investigated over a range 200-600 bar pressure and a shift in reaction rate was noticeable. For this kind of analysis, where the stress and chemical composition depend on each other, the term mechanochemistry has been used in the past [41]. Ramos et al [40] suggested to augment equation (5) and (6) such that

$k_{i}(T, p)=A_{i 0} \exp \left(-\frac{\Delta E_{1}}{T R}\right) \exp \left[\left(\frac{-\Delta V}{T R}+\frac{1}{V} \frac{\partial V}{\partial p}\right)\left(p-p_{0}\right)\right], \quad i=1,2$

where $\Delta V$ is the activation volume and $p$ is the pressure. The dependence of rate of cure on temperature is significantly stronger than on pressure in the range 1-140 bar, which is observed during the curing process in the mold [42-44]. Therefore, the dependence on pressure will be ignored. It is also desirable to separate the thermo-chemical problem from the mechanical 


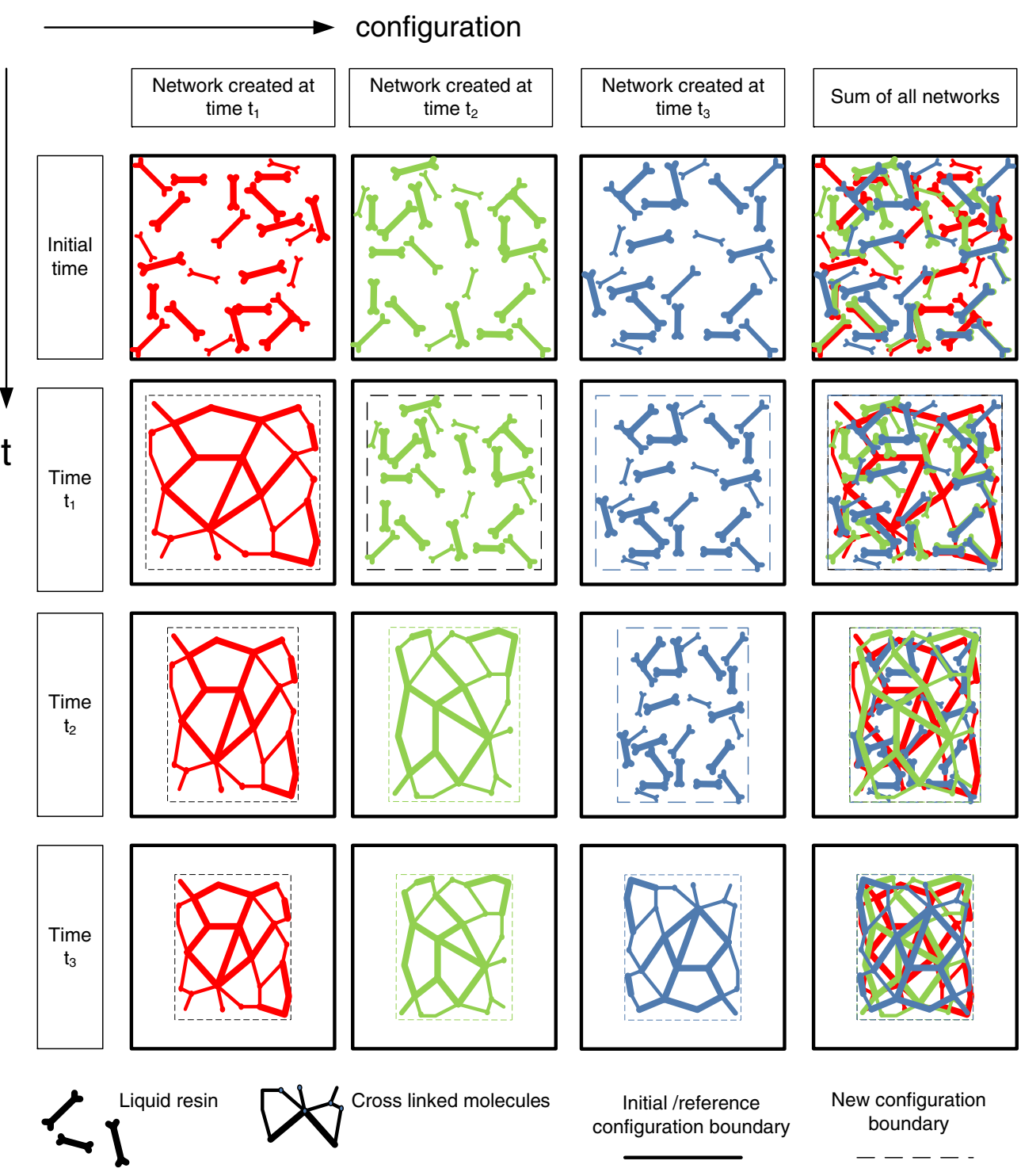

Figure 5. A schematic of the forming of networks as curing evolves.

problem from a numerical point of view. This leads to a one way coupling. The temperature and chemical composition are assumed to be independent of stress state but the stress state depends on the degree of cure through evolving material properties and on temperature through thermal expansion.

In what follows, a model for stress evolution that incorporates the notion of networks forming and contributing to evolving stiffness is derived for a 1D model and then generalized to three dimensions. The creation of the epoxy networks as curing evolves is depicted in figure $5[45,46]$. Initially, hardener and epoxy resin are in a liquid state. They are mixed and poured into a mold, with or without fibers. Immediately after mixing, the epoxy resin starts reacting, forming cross links. At some time $t_{1}$ the first network forms. It is assumed that it forms in a stress-free configuration. Formation of the network is accompanied by some 

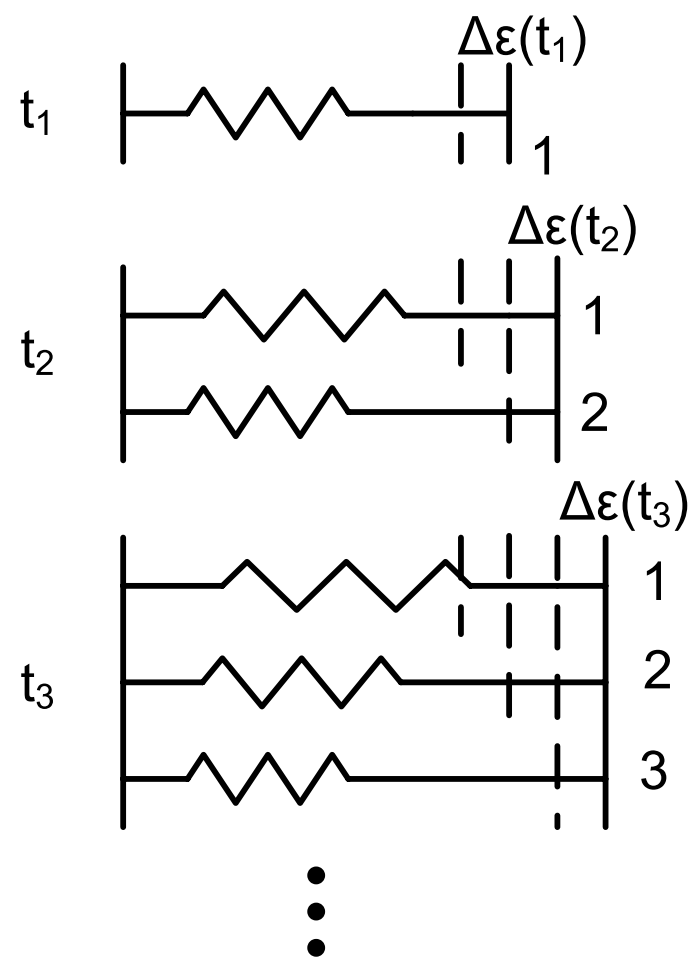

Figure 6. Forming of 1D networks, shown schematically.

shrinkage due to cure. There also can be additional strain due to changes in temperature and externally applied loads. At some later time, $t_{2}$, another network forms. This network has a different stress-free reference configuration. Even later, a new network forms at time $t_{3}$ and this process continues in time. The stress-free reference configuration for the network formed at time $t_{3}$ is different than the one from the networks formed at time $t_{1}$ and $t_{2}$. Because all networks occupy the same volume, their current configuration is the same. On the other hand, their stress-free reference configuration is different. As a result, the stress state in each network is different. The sum of all stresses within the networks is such that it balances out all externally applied resultant forces.

Figure 6 depicts schematically the network formation process using one-dimensional springs. The derivation is similar to the ones given by Mei [46] and Hossain et al [47]. A network forms at time $t_{1}$ and experiences some external strain due to external load. The response of the network is assumed to be elastic. The stress $\sigma(t, s)$ at time $t$ in a network created at time $s$ is proportional to the mechanical strain $\varepsilon^{\text {mech }}(t, s)$ relative to its stress-free reference configuration multiplied by the elastic modulus $E(s)$ associated with that network.

$$
\sigma(t, s)=E(s) \varepsilon^{\text {mech }}(t, s)
$$

The total strain at time $t$ in the network formed at time $s, \varepsilon(t, s)$, is the sum of all incremental strains since its creation. Thus, it is the sum of the mechanical strain, thermal strain and shrinkage strain. $\Delta \varepsilon\left(t_{i}\right)$ is the increment in total strain from time $t_{i-1}$ to time $t_{i}$. Therefore,

$$
\begin{aligned}
\varepsilon(t, s) & =\Delta \varepsilon(s)+\Delta \varepsilon(s+\Delta t)+\cdots+\Delta \varepsilon(s+n \Delta t)+\cdots+\Delta \varepsilon(t) \\
& =\varepsilon^{\text {mech }}(t, s)+\alpha(s) \Delta(T(t)-T(s))-\varepsilon_{\mathrm{c}}(s) .
\end{aligned}
$$


Here, $\varepsilon_{\mathrm{c}}(s)$ is the shrinkage strain of the network formed at time $s$ and $\alpha(s)$ is the coefficient of thermal expansion of that network. The sign of $\varepsilon_{\mathrm{c}}(s)$ is positive when it decreases the strain. The stress in network 1 at time $t_{1}$, when it is created is

$$
\sigma\left(t_{1}, t_{1}\right)=E\left(t_{1}\right)\left(\Delta \varepsilon\left(t_{1}\right)+\varepsilon_{c}\left(t_{1}\right)-\alpha\left(T\left(t_{1}\right)-T\left(t_{1}\right)\right)\right) .
$$

Curing strains begin to impact each network, after it is formed. Strains due to external loads and temperature differences have to be accounted for at all times. The stresses in network 1 and 2 at the formation time of network 2 are given by

$$
\begin{aligned}
& \sigma\left(t_{2}, t_{1}\right)=E\left(t_{1}\right)\left(\Delta \varepsilon\left(t_{1}\right)+\Delta \varepsilon\left(t_{2}\right)+\varepsilon_{c}\left(t_{1}\right)-\alpha\left(T\left(t_{2}\right)-T\left(t_{1}\right)\right)\right), \\
& \sigma\left(t_{2}, t_{2}\right)=E\left(t_{2}\right)\left(\Delta \varepsilon\left(t_{2}\right)+\varepsilon_{c}\left(t_{2}\right)-\alpha\left(T\left(t_{2}\right)-T\left(t_{2}\right)\right) .\right.
\end{aligned}
$$

Furthermore, in the next step, the total strains of the reference configuration change by the amount $\Delta \varepsilon_{3}$ after network 3 is created:

$$
\begin{aligned}
\sigma\left(t_{3}, t_{1}\right)= & E\left(t_{1}\right)\left(\Delta \varepsilon\left(t_{1}\right)+\Delta \varepsilon\left(t_{2}\right)+\Delta \varepsilon\left(t_{3}\right)\right. \\
& \left.+\varepsilon_{c}\left(t_{1}\right)-\alpha\left(T\left(t_{3}\right)-T\left(t_{1}\right)\right)\right), \\
\sigma\left(t_{3}, t_{2}\right)= & E\left(t_{2}\right)\left(\Delta \varepsilon\left(t_{2}\right)+\Delta \varepsilon\left(t_{3}\right)+\varepsilon_{c}\left(t_{2}\right)-\alpha\left(T\left(t_{3}\right)-T\left(t_{2}\right)\right)\right), \\
\sigma\left(t_{3}, t_{3}\right)= & E\left(t_{3}\right)\left(\Delta \varepsilon\left(t_{3}\right)+\varepsilon_{c}\left(t_{3}\right)-\alpha\left(T\left(t_{3}\right)-T\left(t_{3}\right)\right)\right) .
\end{aligned}
$$

Each network is weighted to contribute to the total load carrying capability in a 'rule of mixtures' sense. During the creation of each network, the overall epoxy has cured by an amount $\Delta \phi\left(t_{i}\right)=\phi\left(t_{i}\right)-\phi\left(t_{i-1}\right)$, where, $\phi$ denotes the degree of cure as defined by equation (1). In the following, it will be assumed that an equivalent interpretation of the degree of cure $\phi$ is given by

$$
\begin{aligned}
& \begin{aligned}
\phi(t) & =\frac{\text { mass cured at time } t}{\text { total mass of material that can cure }} \\
& =\text { mass fraction of cured material, }
\end{aligned} \\
& \mathrm{d} \phi=\text { mass fraction cured during the time interval from } s \text { to } s+\mathrm{d} s .
\end{aligned}
$$

The total stress in the epoxy at time $t_{N}$ is given by

$$
\begin{aligned}
\sigma_{\mathrm{tot}}\left(t_{N}\right)=\sum_{i=1}^{N} & \Delta \phi\left(t_{i}\right) \sigma\left(t_{N}, t_{i}\right)=\sum_{i=1}^{N} \Delta \phi\left(t_{i}\right) E\left(t_{i}\right) \\
& \times\left(\varepsilon_{c}\left(t_{i}\right)-\alpha\left(T\left(t_{N}\right)-T\left(t_{i}\right)\right)+\sum_{j=i}^{N} \Delta \varepsilon\left(t_{N}, t_{j}\right)\right) .
\end{aligned}
$$

The tot subscript will be omitted in the following, when it is clear that the total stress is meant. When taking the limit of the above equation, the sums can be converted into integrals:

$$
\sum \Delta \phi \rightarrow \int \mathrm{d} \phi \quad \text { and } \quad \sum \Delta \varepsilon \rightarrow \int \mathrm{d} \varepsilon .
$$

The discrete quantity $\varepsilon_{\mathrm{c}}\left(t_{i}\right)$ represents the shrinkage strain in the $i$ th network formed at time $t_{i}$. Its continuous equivalent is $\varepsilon_{\mathrm{c}}(s)$. Equivalent expressions exist for the material properties $E\left(t_{N}\right) \rightarrow E(s)$ and $\alpha\left(t_{N}\right) \rightarrow \alpha(s)$. With these substitutions, the stress up to a certain amount of cure, represented by the cure fraction, $\Phi$ is given by

$$
\begin{aligned}
\sigma(\Phi) & =\int_{0}^{\Phi} \tilde{E}(\phi)\left(\varepsilon_{\mathrm{c}}(\phi)-\alpha(T(\Phi)-T(\phi))+\int_{\varepsilon(\phi)}^{\varepsilon(\Phi)} \mathrm{d} \bar{\varepsilon}\right) \mathrm{d} \phi \\
& =\int_{0}^{\Phi} \tilde{E}(\phi)\left(\varepsilon_{\mathrm{c}}(\phi)-\alpha(T(\Phi)-T(\phi))+\varepsilon(\Phi)-\varepsilon(\phi)\right) \mathrm{d} \phi .
\end{aligned}
$$


The degree of cure $\phi(s)$ increases monotonically with time which allows for the substitution, $\mathrm{d} \phi=(\mathrm{d} \phi / \mathrm{d} s) \mathrm{d} s$ :

$$
\sigma(t)=\int_{0}^{t} \frac{\mathrm{d} \phi}{\mathrm{d} s} E(s)\left(\varepsilon(t)-\varepsilon(s)+\varepsilon_{\mathrm{c}}(s)-\alpha(T(t)-T(s))\right) \mathrm{d} s .
$$

The elastic modulus $E(s)$ depends directly on the degree of cure, and indirectly on time, by $E(s)=\tilde{E}(\phi(s))$. The above equation can be generalized to three dimensions:

$$
\begin{aligned}
\underline{\underline{\sigma}}= & \int_{0}^{t} \frac{\mathrm{d} \phi}{\mathrm{d} s}\left[\underline{\underline{1}} K(s) \operatorname{tr}\left(\underline{\underline{\varepsilon}}(t)-\underline{\underline{\varepsilon}}(s)+\underline{\underline{\varepsilon_{\mathrm{c}}}}(s)-\underline{\underline{1}} \alpha(s) \Delta T(t, s)\right)\right. \\
& \left.+2 \mu(s)\left(\underline{\underline{\varepsilon}}(t)-\underline{\underline{\varepsilon}}(s)+\underline{\underline{\varepsilon_{\mathrm{c}}}}(s)-\underline{\underline{1}} \frac{1}{3} \operatorname{tr}\left(\underline{\underline{\varepsilon}}(t)-\underline{\underline{\varepsilon}}(s)+\underline{\underline{\varepsilon_{\mathrm{c}}}}(s)\right)\right)\right] \\
& +(1-\phi(t)) K_{\mathrm{liq}} \operatorname{tr}\left(\underline{\underline{\varepsilon}}(t)-\underline{\underline{1}} \alpha_{\mathrm{liq}} \Delta T(t)\right) \underline{\underline{1}},
\end{aligned}
$$

where $K$ is the per-network bulk modulus, $\mu$ is the per-network shear modulus and $\alpha$ is the linear coefficient of thermal expansion of each network. $K_{\text {liq }}$ and $\alpha_{\text {liq }}$ are the bulk modulus and coefficient of thermal expansion of the uncured liquid epoxy resin. The notation, (.) indicates a second order tensor. The last term represents the contribution to the total stress from the volume fraction of uncured epoxy liquid. The liquid can hold compressive stress but its shear stress is neglected.

The tensor quantity $\underline{\underline{\varepsilon_{\mathrm{c}}}}(s)$ describes the cure shrinkage strains of each network. It is assumed that curing only produces equal normal strains:

$$
\underline{\underline{\varepsilon_{\mathrm{c}}}}(s)=\left[\begin{array}{ccc}
\varepsilon_{\mathrm{c}}(s) & 0 & 0 \\
0 & \varepsilon_{\mathrm{c}}(s) & 0 \\
0 & 0 & \varepsilon_{\mathrm{c}}(s)
\end{array}\right]
$$

$\underline{\underline{\varepsilon_{\mathrm{c}}}}(s)$ can be chosen such that macroscopically, shrinkage as a linear function of degree of cure can be observed.

It is noted that formally $\alpha(s), \mu(s), K(s)$ are functions of time. But actually they are a function of cure, which has to be related to time, so that $\alpha(s)=\tilde{\alpha}(\phi(s)), \mu(s)=\tilde{\mu}(\phi(s))$, $K(s)=\tilde{K}(\phi(s))$ and $\varepsilon_{\mathrm{c}}(s)=\tilde{\varepsilon}_{c}(\phi(s))$. These material functions are determined from experiments.

\section{Mechanical properties}

\subsection{Elastic properties}

The longitudinal modulus, shear modulus and degree of cure are measured as a function of time through concurrent Raman (RLS) and Brillouin (BLS) light scattering [48-53]. The longitudinal modulus $M$ is the elastic constant measured in a uniaxial strain field. It is related to the Lamé constants and the bulk modulus by $M=\lambda+2 \mu=K+\frac{4}{3} \mu$. Raman scattering provides cure information and Brillouin light scattering provides mechanical information. Furthermore, the measurements are carried out concurrently, i.e. the signals for both measurements were collected at the same time from the same focal volume of the probing laser beam. These optical measurement methods have the advantage of yielding mechanical and chemical properties nondestructively and without perturbing the thermodynamic equilibrium of the probed system. In contrast, classical methods that measure the mechanical properties during cure, such as rheometers or bending tests [54], actively influence the BVP that is needed to extract the per-network properties for this approach. 
It is important to note that experimentally, the response of all networks is measured simultaneously. The individual response of each network has to be adjusted so that the total simulated response follows the total experimentally measured response. To this end, assume that the network is cured in a uniaxial strain field. After some time, a sudden jump in strain is applied. The jump needs to be considered for all times during the curing history, because the material properties need to be extracted for all times during the curing history. The stress response is measured in the direction the strain jump is applied, and in the perpendicular direction.

The strain tensor of the uniaxial strain field, before and after a small increment in strain are

$$
\underline{\varepsilon}=\left[\begin{array}{ccc}
\varepsilon & 0 & 0 \\
0 & 0 & 0 \\
0 & 0 & 0
\end{array}\right]
$$

and,

$$
\underline{\underline{\varepsilon}}=\left[\begin{array}{ccc}
\varepsilon+\Delta \varepsilon & 0 & 0 \\
0 & 0 & 0 \\
0 & 0 & 0
\end{array}\right],
$$

respectively. The strain increment is applied instantaneously. Therefore no variation in temperature takes place during this event. The stresses before and after the application of the strain increment are

$$
\begin{aligned}
& \underline{\underline{\sigma}}=\left[\begin{array}{ccc}
\sigma_{11} & 0 & 0 \\
0 & \sigma_{22} & 0 \\
0 & 0 & \sigma_{33}=\sigma_{22}
\end{array}\right], \\
& \underline{\underline{\sigma}}+\Delta \underline{\underline{\sigma}}=\left[\begin{array}{ccc}
\sigma_{11}+\Delta \sigma_{11} & 0 & 0 \\
0 & \sigma_{22}+\Delta \sigma_{22} & 0 \\
0 & 0 & \sigma_{33}=\sigma_{22}+\Delta \sigma_{22}
\end{array}\right] .
\end{aligned}
$$

Thus,

$$
\begin{gathered}
\sigma_{11}=\int_{0}^{t} \dot{\phi}\left\{K\left[\varepsilon(t)-\varepsilon(s)+3 \varepsilon_{\mathrm{c}}(s)\right]+\frac{4}{3} \mu[\varepsilon(t)-\varepsilon(s)]\right\} \mathrm{d} s+(1-\phi(t)) K_{\mathrm{liq}} \varepsilon(t), \\
\sigma_{11}+\Delta \sigma_{11}=\int_{0}^{t} \dot{\phi}\left\{K\left[\varepsilon(t)+\Delta \varepsilon-\varepsilon(s)+3 \varepsilon_{\mathrm{c}}(s)\right]+\frac{4}{3} \mu[\varepsilon(t)+\Delta \varepsilon-\varepsilon(s)]\right\} \mathrm{d} s \\
+(1-\phi(t)) K_{\mathrm{liq}}(\varepsilon(t)+\Delta \varepsilon) .
\end{gathered}
$$

The difference between the two is

$$
\begin{aligned}
\Delta \sigma_{11} & =\Delta \varepsilon \int_{0}^{t} \dot{\phi}\left\{K(s)+\frac{4}{3} \mu(s)\right\} \mathrm{d} s+\Delta \varepsilon(1-\phi) K_{\text {liq }} \\
& =\Delta \varepsilon \int_{0}^{t} \dot{\phi} M(s) \mathrm{d} s+\Delta \varepsilon(1-\phi) K_{\text {liq }} .
\end{aligned}
$$

Thus, the incremental stress-strain relations can be written as

$$
\Delta \sigma_{11}=\Delta \varepsilon_{11} M_{\mathrm{tot}}(t)
$$

where

$$
\begin{aligned}
M_{\mathrm{tot}} & =\int_{0}^{t} \dot{\phi} M(s) \mathrm{d} s+(1-\phi(t)) K_{\mathrm{liq}} \\
& =\int_{0}^{t} \dot{\phi}\left\{K(s)+\frac{4}{3} \mu(s)\right\} \mathrm{d} s+(1-\phi(t)) K_{\mathrm{liq}}
\end{aligned}
$$




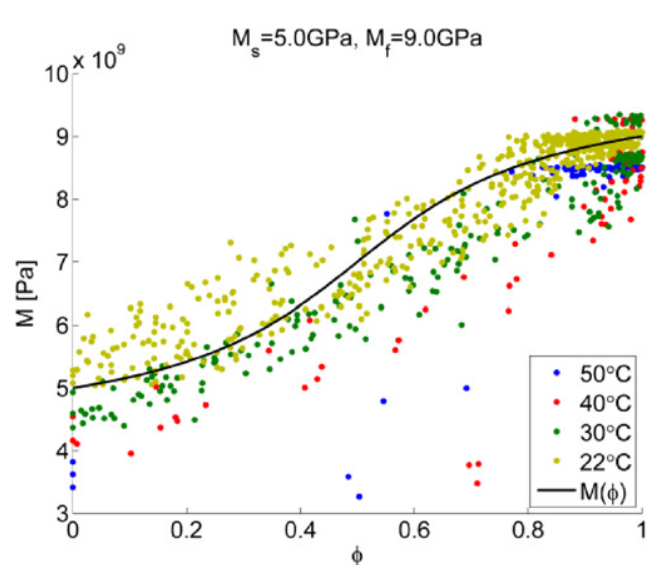

(a) Longitudinal modulus

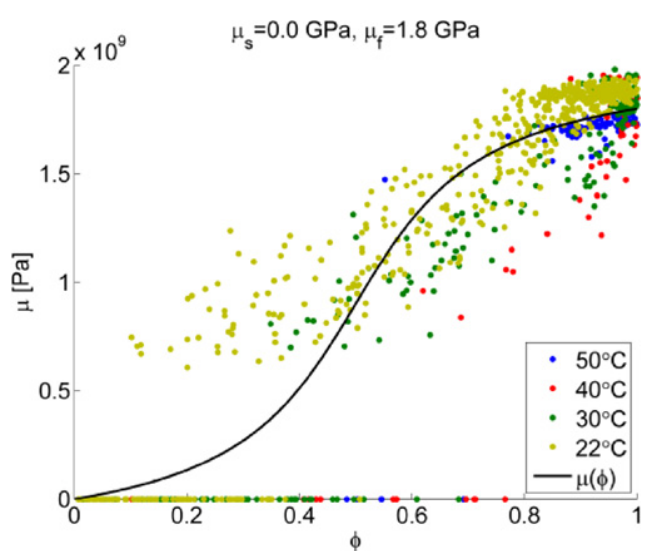

(b) Longitudinal modulus

Figure 7. Experimental results and curve fit of evolution of elastic properties as a function of cure obtained through Brillouin and Raman light scattering.

is the total longitudinal modulus, and $M(s)$ is the plane wave modulus of each network formed at time $s$. Differentiating with respect to time, $t$, rearranging and applying the chain rule yields the per-network properties.

$$
M(\phi)=\frac{\mathrm{d} M_{\mathrm{tot}}(\phi)}{\mathrm{d} \phi}+K_{\text {liq }} .
$$

The same BVP can be used to determine a second set of properties. For the direction perpendicular to the one in which the strain is applied, it follows that

$$
\begin{aligned}
\Delta \sigma_{22} & =\Delta \varepsilon \int_{0}^{t} \dot{\phi}\left(K(s)-\frac{2}{3} \mu(s)\right) \mathrm{d} s+\Delta \varepsilon(1-\phi) K_{\text {liq }} \\
& =\Delta \varepsilon \int_{0}^{t} \dot{\phi} \lambda(s) \mathrm{d} s+\Delta \varepsilon(1-\phi) K_{\text {liq }} .
\end{aligned}
$$

For this particular BVP,

$$
\Delta \sigma_{22}=\Delta \varepsilon_{11} \lambda_{\text {tot }}(t)
$$

where

$$
\lambda_{\text {tot }}=\int_{0}^{t} \dot{\phi}(s) \lambda(s) \mathrm{d} s+(1-\phi(t)) K_{\text {liq }}
$$

so that the per-network Lamé constant is

$$
\lambda(\phi)=\frac{\mathrm{d} \lambda_{\text {tot }}(\phi)}{\mathrm{d} \phi}+K_{\text {liq }}
$$

This can be transformed to yield the per-network shear and bulk moduli:

$$
\begin{aligned}
& \mu(\phi)=\frac{\mathrm{d} \mu_{\mathrm{tot}}}{\mathrm{d} \phi}, \\
& K(\phi)=\frac{\mathrm{d} K_{\mathrm{tot}}}{\mathrm{d} \phi}+K_{\text {liq }} .
\end{aligned}
$$

Figures 7( $a$ ) and $(b)$ show representative experimental data for $M$ and $\mu$ as functions of the extent of cure of a Epon $862^{\circledR}$ sample. The longitudinal modulus, shear modulus and degree of 
cure are measured as a function of time through concurrent Raman (RLS) and Brillouin (BLS) light scattering [48-53]. The longitudinal modulus $M$ is the elastic constant measured in a uniaxial strain field. Raman scattering provides cure information and Brillouin light scattering provides mechanical information.

For mathematical convenience the transition of these material properties is currently modeled analytically:

$$
\begin{aligned}
& \mu_{\mathrm{tot}}(\phi)=\frac{\arctan \left(\left(\phi-\frac{1}{2}\right) \beta_{\mu}\right)}{\arctan \left(\beta_{\mu} / 2\right)}\left(\mu_{\mathrm{f}}-\mu_{\mathrm{s}}\right)+\left(\frac{\mu_{\mathrm{f}}+\mu_{\mathrm{s}}}{2}\right), \\
& M_{\mathrm{tot}}(\phi)=\frac{\arctan \left(\left(\phi-\frac{1}{2}\right) \beta_{M}\right)}{\arctan \left(\beta_{M} / 2\right)}\left(M_{\mathrm{f}}-M_{\mathrm{s}}\right)+\left(\frac{M_{\mathrm{f}}+M_{\mathrm{s}}}{2}\right),
\end{aligned}
$$

where $\mu_{\mathrm{s}}, \mu_{\mathrm{f}}, M_{\mathrm{s}}, M_{\mathrm{f}}$ are the start and final value of the measure shear modulus and longitudinal modulus, respectively. $\beta_{\mu}$ and $\beta_{M}$ are fitting constants of the experimental data, related to the curvature of the experimental fit. This leads to closed form solutions for the per-network properties:

$$
\begin{aligned}
\mu(\phi) & =\frac{1}{2} \frac{\beta_{\mu}\left(\mu_{\mathrm{f}}-\mu_{\mathrm{s}}\right)}{\left(1+(\phi-1 / 2)^{2} \beta_{\mu}^{2}\right) \arctan \left(1 / 2 \beta_{\mu}\right)} \\
M(\phi) & =\frac{1}{2} \frac{\beta_{M}\left(M_{\mathrm{f}}-M_{\mathrm{s}}\right)}{\left(1+(\phi-1 / 2)^{2} \beta_{M}^{2}\right) \arctan \left(1 / 2 \beta_{M}\right)}+K_{\text {liq }} \\
K(\phi) & =M(\phi)-\frac{4}{3} \mu(\phi) .
\end{aligned}
$$

In the curing model, a small amount of shear stiffness is assumed even for low degrees of cure, as shown in figure $7(b)$. This is necessary for two reasons: as can be seen from equation (44) a discontinuity in the total shear modulus would lead to a singularity of the per-network shear modulus in the context of the proposed curing model. Secondly, the finite element procedure uses solid elements that require a non-zero shear stiffness. On the other hand, it should be noted that in reality the epoxy behaves like a viscous liquid until gelation occurs. It is only from that moment onwards that a finite shear modulus is detected. During the simulations it was found that despite the assumption of a small amount of shear modulus for a low degree of cure, no appreciable stress level was detected during the early phase of the curing process.

Finally, the bulk modulus of the liquid epoxy is the one measured at the beginning of the experiment.

$$
K_{\text {liq }}=K_{\text {tot }}(0)
$$

\subsection{Cure shrinkage}

To determine the correct cure shrinkage, a BVP needs to be formulated, where the change in volume can be measured. Therefore, consider again a uniaxial strain BVP of a curing epoxy sample. The specimen is free to expand or contract in the 1-direction. No stresses are applied in this direction. The stress and strain tensors are

$$
\underline{\underline{\sigma}}=\left[\begin{array}{ccc}
\sigma_{11}=0 & 0 & 0 \\
0 & \sigma_{22} & 0 \\
0 & 0 & \sigma_{33}=\sigma_{22}
\end{array}\right]
$$

and

$$
\underline{\underline{\varepsilon}}=\left[\begin{array}{lll}
\varepsilon & 0 & 0 \\
0 & 0 & 0 \\
0 & 0 & 0
\end{array}\right]
$$


The stress in the 1-direction is given by

$\sigma_{11}=\int_{0}^{t} \dot{\phi}(s) M(s)(\varepsilon(t)-\varepsilon(s)) \mathrm{d} s+3 \int_{0}^{t} \varepsilon_{\mathrm{c}}(s) K(s) \dot{\phi}(s) \mathrm{d} s+(1-\phi(t)) \varepsilon(t) K_{\text {liq }}$,

and it is equal to zero. There is a monotonic relation between time, $t$, and degree of cure, $\phi$. A change in variables of $t \rightarrow \Phi=\phi(t)=$ and $\dot{\phi} \mathrm{d} s \rightarrow \mathrm{d} \phi$ leads to

$\sigma_{11}(\Phi)=\int_{0}^{\Phi} M(\phi)(\varepsilon(\Phi)-\varepsilon(\phi)) \mathrm{d} \phi+3 \int_{0}^{\Phi} \varepsilon_{\mathrm{c}}(\phi) K(\phi) \mathrm{d} \phi+(1-\Phi) \varepsilon(\Phi) K_{\text {liq }}=0$.

Differentiating with respect to the upper limit, observing that no stress was applied in the 1-direction and rearranging, yields the final expression for the cure shrinkage in terms of the strain in the direction of the free surface;

$\varepsilon_{\mathrm{c}}(\Phi)=\frac{1}{3 K(\Phi)}\left[\left(\varepsilon(\Phi)-(1-\Phi) \frac{\mathrm{d} \varepsilon(\Phi)}{\mathrm{d} \Phi}\right) K_{\text {liq }}-\frac{\mathrm{d} \varepsilon(\Phi)}{\mathrm{d} \Phi} \int_{0}^{\Phi} M(\phi) \mathrm{d} \phi\right]$.

The total cure shrinkage of all networks $\varepsilon(\Phi)$ can be measured through a gravimetric test method [55]. In view of similar epoxy systems [56,57] a cure shrinkage of $2 \%$ has been chosen for this study.

\section{Numerical modeling}

The curing problem was solved with the commercial finite element code Abaqus/Standard [58] with the help of Fortran user subroutines. These problems are solved in sequence. First the evolution of temperature and chemistry is solved using user-defined heat generation (HETVAL). Next a 'static step' is used to compute the evolution of mechanical properties and stress. Here a user-defined material subroutine (UMAT) is used. For these types of problems the numerical implementation is extremely robust and efficient. The majority of the elements used were quadratic hexahedrals (C3D20). When necessary, quadratic wedge elements (C3D15) were inserted to avoid mesh distortion.

The boundary conditions in the transverse direction were periodic [23]. Generalized plane strain boundary conditions have been used in the axial direction. The RVE was able to expand and contract in that direction. The carbon fibers are modeled as transversely isotropic.

$$
\begin{aligned}
& u_{1}\left(L_{1}, x_{2}\right)-u_{1}\left(0, x_{2}\right)=E_{11} L_{1}, \\
& u_{2}\left(L_{1}, x_{2}\right)-u_{2}\left(0, x_{2}\right)=0, \\
& u_{1}\left(x_{1}, L_{2}\right)-u_{1}\left(x_{1}, 0\right)=2 E_{12} L_{2}, \\
& u_{2}\left(x_{1}, L_{2}\right)-u_{1}\left(x_{1}, 0\right)=E_{22} L_{2},
\end{aligned}
$$

where, $E_{i j}$, are the macroscopically measured/homogenized strains of the RVE and $L_{1}$ and $L_{2}$ are the side lengths of the RVE in the 1 and 2 direction, respectively. The boundary conditions are also depicted in figure 8 . The boundary conditions in the 3 direction where chosen such that plane sections remain plane, i.e. the displacement in the 3 direction is the same everywhere at any given height. The macroscopic strains were not prescribed. Instead, the master nodes associated with the right-hand side in equation (57)-(60) had no force associated with them. This is equivalent to a stress-free boundary condition. The unit cell is considered small enough such that gradients in temperature are small. Therefore the temperature was prescribed to be the same at every material point. The epoxy was initially at room temperature at $293 \mathrm{~K}$ and then quickly ramped to $333 \mathrm{~K}$ and kept there until full cure. When considering a composite part on the macroscopic level, i.e. the tow level, heat conduction has to be taken into account. 

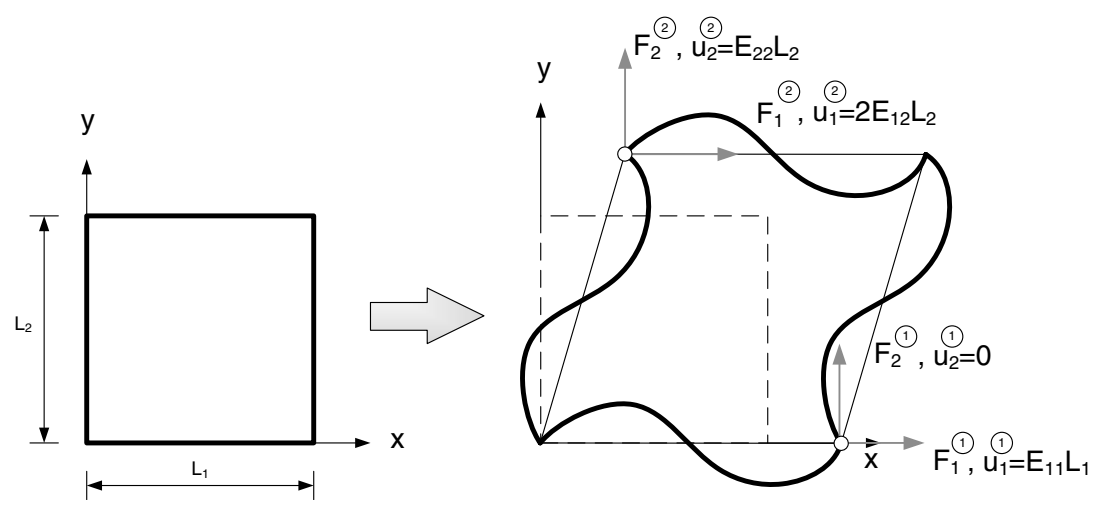

Figure 8. Periodic boundary conditions.

For a specified amount of randomness and volume fraction, 750 unit cells have been sampled over a range 5-50 fibers each. The amount of randomness is chosen to be described by the relative standard deviation of the Voronoi cells relative to the average size, i.e.

$$
\bar{\sigma}=\sqrt{\frac{1}{n} \sum_{i=1}^{n}\left(A_{i}-\bar{A}\right)^{2}} / \bar{A},
$$

where $A_{i}$ is the area of each Voronoi polygon and $\bar{A}$ is the average area of the Voronoi polygons. The Voronoi cells have been generated numerically. Only the relative standard deviation of the Voronoi cells relative to the average size has been chosen as a metric of randomness and on that basis the microstructure was generated.

The RVE generation problem is treated as a molecular dynamics type simulation. The fibers are placed as particles in a random manner with large repulsion if they get too close to each other (i.e. the centers are less than two fiber radii away from each other) to prevent overlapping and slight attraction if they are farther apart. The randomness is included by introducing additional 'charges' that repel all fibers by a certain varying amount. The motivation for introducing these 'charges' is that they represent matrix rich regions in real microstructures found in textile composites. By varying the number and strength of these repulsive 'charges' the randomness can be dialed in quite precisely. Due to the 'forces' between the points that represent the fibers, and a resulting self-rearrangement, large fiber-volume fractions can be obtained without the jamming that is found in hard core models [30].

The convergence of stresses in the curing unit cell as a function of fiber number has been compared to a similar RVE subjected to shear loading. It is to be noted that the latter BVP solution does not include the effects of the curing stresses. The shear loading BVP simulations were done in the context of plane strain elastic-plastic behavior. Whenever possible, quadratic plane strain elements were used with quadratic triangles inserted whenever necessary to avoid mesh distortion. The material properties were comparable to carbon fiber and Epon ${ }^{\circledR}$ epoxy. The matrix material properties used for the curing simulations are given in table 1 and the fiber properties are given in table 2.

A mesh convergence study has been performed on a typical unit cell that contains 5 fibers. The results are given in figure 9. For the shear response, a typical element size of $0.5 \mu \mathrm{m}$ has been used for further simulations. This corresponds to 1800 elements in a 5-fiber RVE. A typical element size of $1.0 \mu \mathrm{m}$ has been used for all curing simulations. This corresponds to 900 elements in a 5-fiber RVE. These element sizes show sufficient convergence while still 
Table 2. Material properties of carbon fiber (1 is the fiber direction) [59].

\begin{tabular}{ll}
\hline Coefficient of thermal expansion $\alpha_{11}$ & $-0.4 \times 10^{-6}$ \\
Coefficient of thermal expansion $\alpha_{22}$ & $5.6 \times 10^{-6}$ \\
Elastic modulus $E_{1}$ & $276 \mathrm{GPa}$ \\
Elastic modulus $E_{2}$ & $19.5 \mathrm{GPa}$ \\
Shear modulus $G 13$ & $70 \mathrm{GPa}$ \\
Shear modulus $G 23$ & $5.735 \mathrm{GPa}$ \\
Poisson's ratio $v_{13}$ & 0.28 \\
Poisson's ratio $v_{23}$ & 0.7 \\
\hline
\end{tabular}

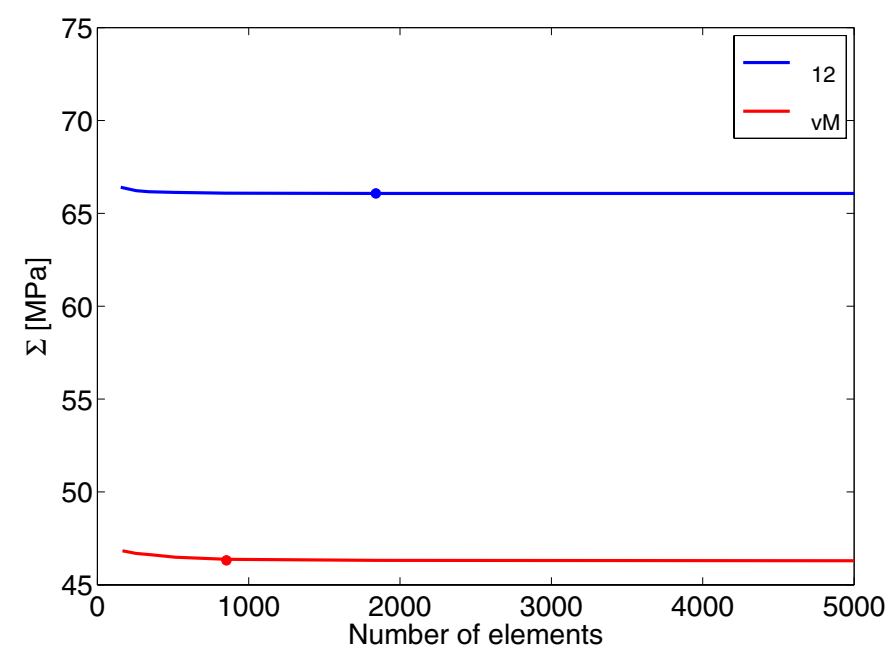

Figure 9. Mesh convergence of 5-fiber unit cell for shear stress and curing stress.

being computationally efficient when using RVEs that contain more fibers. The two simulation types are explained in detail below.

\section{Results and discussion}

Two different simulation cases will be discussed in the following: shear load of a unit cell and cure of a unit cell. These simulation cases will be discussed in view of five different geometric arrangements that differ in measure of randomness, $\bar{\sigma}$, and fiber-volume fraction. All cases under consideration are presented in table 3.

Representative results for case AS for the shear response of a unit cell with 5 fibers (random), 50 fibers (random) and 2 fibers (hexagonal packing) are given in figure 10. The random unit cells had a 'randomness' of $\bar{\sigma}=10 \%$ according to equation (61) and the fiber-volume fraction was $50 \%$. In all cases, the unit cells were sheared to a macroscopic strain $E_{12}=0.02$. The average stresses and strains were calculated by summing the stresses and strains at the integration points [60]. They were then normalized by the total volume:

$$
\begin{aligned}
& \Sigma_{i j}=\frac{1}{V} \int_{V} \sigma_{i j} \mathrm{~d} V, \\
& E_{i j}=\frac{1}{V} \int_{V} \epsilon_{i j} \mathrm{~d} V .
\end{aligned}
$$


Table 3. Simulation cases investigated.

\begin{tabular}{llll}
\hline Case name & $\begin{array}{l}\text { Simulation } \\
\text { type }\end{array}$ & $\begin{array}{l}\text { Randomness } \\
(\%)\end{array}$ & $\begin{array}{l}\text { Fiber-volume } \\
\text { fraction }(\%)\end{array}$ \\
\hline AS & Shear & 10 & 50 \\
AC & Cure & 10 & 50 \\
BS & Shear & 5 & 50 \\
BC & Cure & 5 & 50 \\
CS & Shear & 15 & 50 \\
CC & Cure & 15 & 50 \\
DS & Shear & 10 & 42.5 \\
DC & Cure & 10 & 42.5 \\
ES & Shear & 10 & 35 \\
EC & Cure & 10 & 35 \\
\hline
\end{tabular}
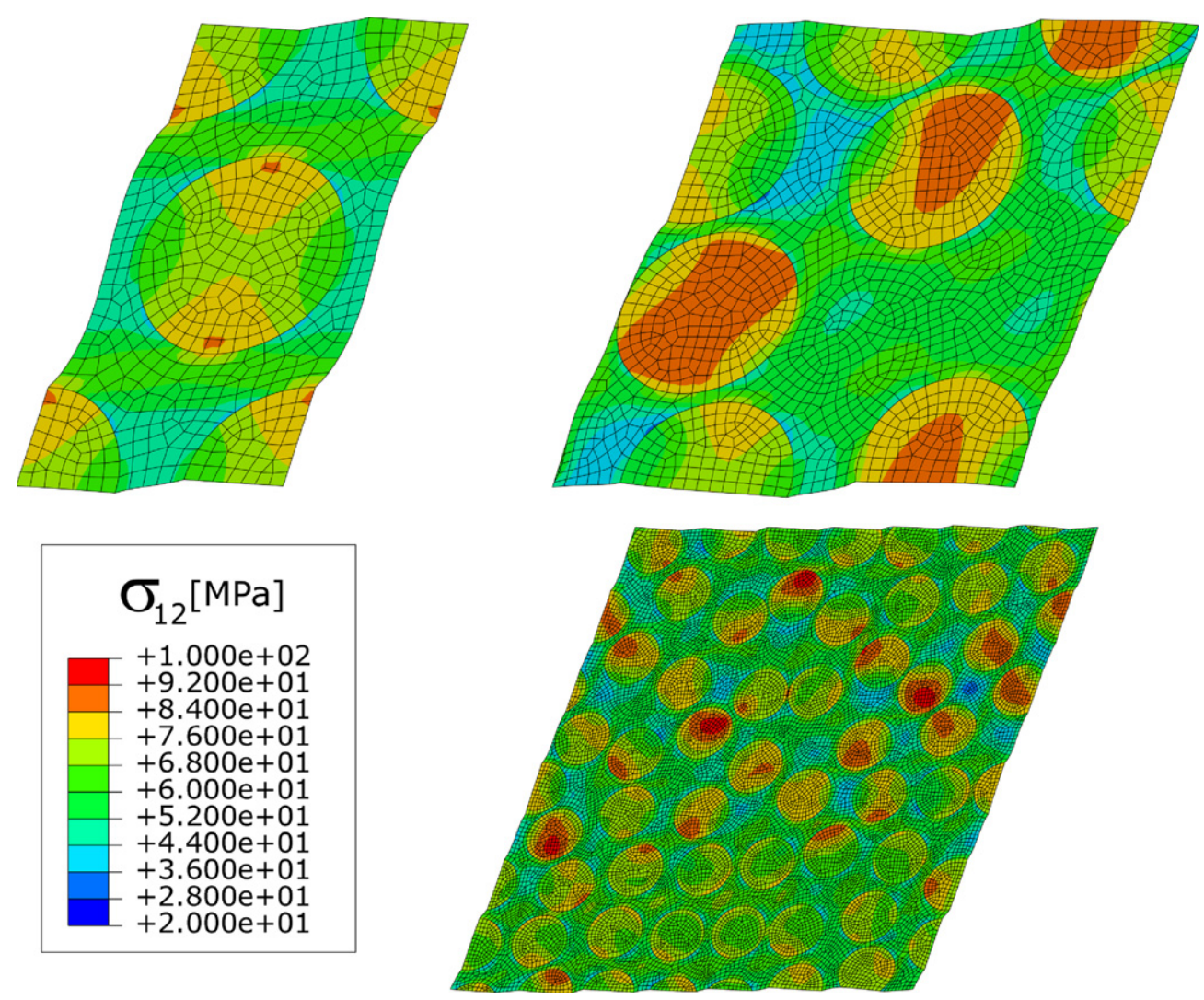

Figure 10. Shear response of hexagonal, 5-fiber and 50-fiber unit cell.

It is worth noting that the average shear strain $E_{12}$ calculated using equation (63) is indeed identical to the strain imposed by the periodic boundary conditions (57)-(60). In general, the average shear stress is largest in the the smallest random unit cell, see figure 12. With increasing unit cell size and fiber number the average shear stress decreases in the random unit cells. The hexagonal packing marks a lower bound for the response. This behavior is somewhat expected. Compared with a hexagonal packing arrangement, random packing 
places some fibers closer to each other. This causes stress concentrations and earlier yielding in these regions compared to the overall unit cell. On the other hand, there are also larger homogeneous regions or matrix rich regions. Here stress concentrations are lowered and yield is delayed. Due to the non-linear nature of the problem the reduced stresses in regions of less fibers combined with the increased stresses in regions of closer fibers do not reproduce the same results as regularly dispersed fibers. From a statistical point of view the deviation in responses between different samples of the same unit cell size decreases with increasing fiber number. For larger samples there are still regions where fibers are close to each other but for the same measure of randomness these regions of high stress appear less often, than for small samples. It is interesting to note that in the range of values investigated, the results for macroscopic values are fairly insensitive to the amount of randomness applied. For case AS, BS and CS the macroscopic shear stress approaches $65 \mathrm{MPa}$ with increasing fiber numbers. From cases AS, DS and ES it can be seen that a decrease in fiber-volume fraction causes a decrease in the relative error between random packing and hexagonal packing. These results have implications for analyses related to damage and failure predictions since extreme properties dictate the onset of damage and failure, whereas, average values can be used for stiffness predictions. In particular, the need to use RVEs that contain multiple fibers and reflect the statistical aspects of the composite material is recommended for computational schemes that adopt multiscale features.

Now, similar observations are made for the cured composite. A typical stress distribution after cure for a 5-fiber RVE, 50-fiber RVE and for hexagonal packing is shown in figure 11. The average curing stress vs. fibers in a RVE are shown in figure 13. The residual von Mises stresses are largest in small random unit cells and decrease when more fibers are added. Also, the spread between different samples of the same random unit cell size decreases with increasing amount of fibers. The hexagonal packing produces the lowest residual stresses. A reduction in randomness causes a decrease in curing stresses as shown in cases AC, BC and $\mathrm{CC}$. Also a reduction in fiber-volume fraction causes a reduction in relative error between hexagonal response and random packing. For case EC the average von Mises stress converges quickly, albeit to a value lower, then the hexagonal packing.

For the curing case, as well as the shear loading case, the largest variations in simulation outcomes can be found for unit cells that are smaller in size than 25 fibers. On the other hand there is still some variation in the response even for unit cells that contain up to 50 fibers. The typical size of a fiber strand in a textile braided composite is on the order of thousands to ten thousands of fibers. However, it would be prohibitively expensive to model all fibers explicitly and it would be much more desirable to use a reduced amount of fibers that yield almost the same response. It should also be kept in mind that in the case of the curing model used here, only residual stress built-up is investigated. The response of such a fully cured unit cell to a subsequent shear load requires a proper damage and failure model which are the subject of a different study (see, [32,33]). It is noted that even for the hexagonal packing case, the residual stresses are on average $45 \mathrm{MPa}$. These were determined for a cure shrinkage of $2 \%$. Hetron ${ }^{\circledR}$ polymer, as studied in [61], has a cure shrinkage of approximately $7 \%$. The stresses will increase correspondingly and finally lead to cracks in the cured part as seen in figure 4 . The formation of unintended damage is believed to be a mechanism that leads to the softening in effective material properties in the manufactured part as described in [12].

\section{Concluding remarks}

The influence of the representative volume element (RVE) size on the residual stresses created during the curing process of a continuous fiber-reinforced polymer matrix composite tow 

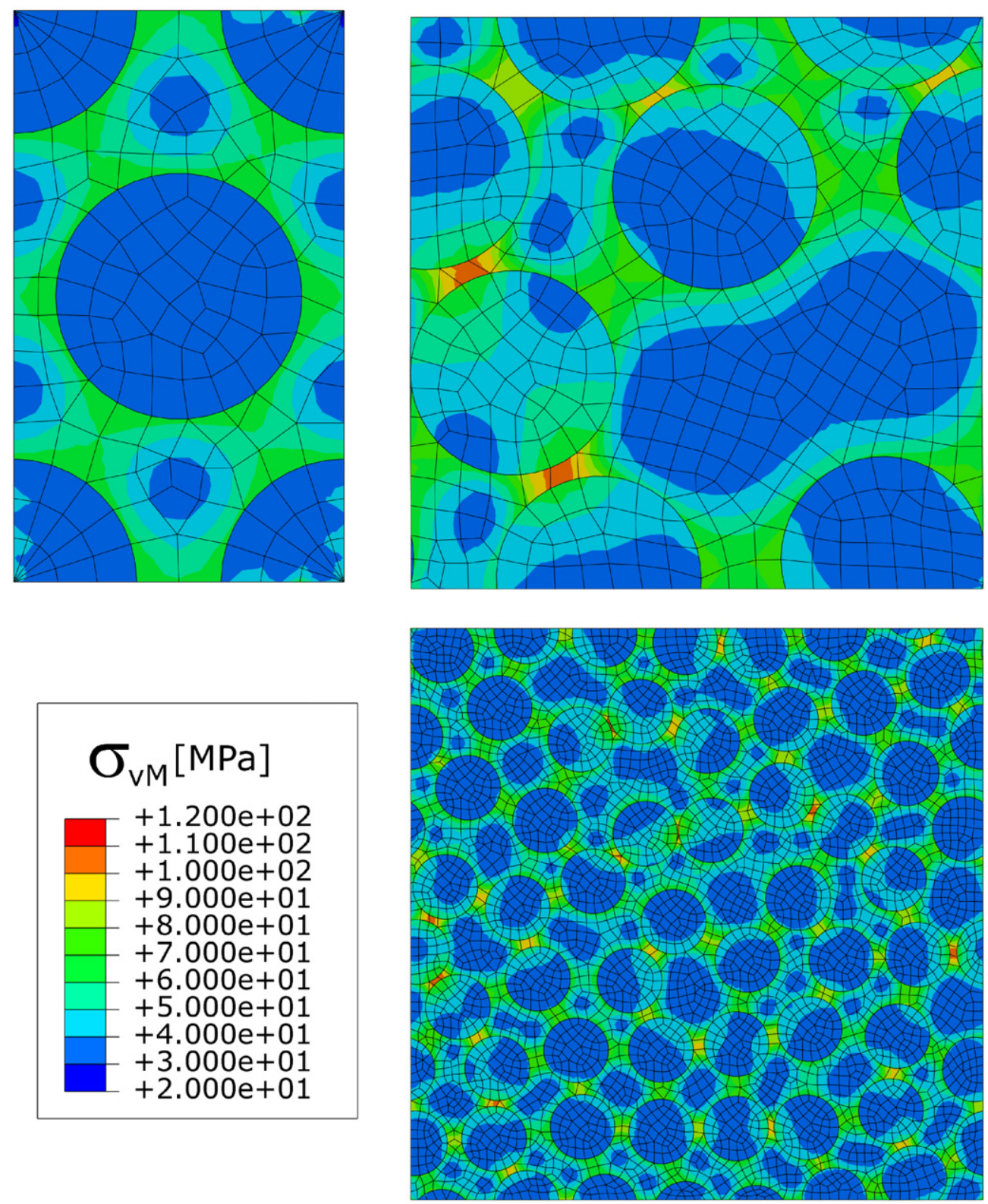

Figure 11. Residual stresses due to cure in hexagonal, 5-fiber and 50-fiber unit cell.

(PMCs) was investigated using a novel network curing model to simulate the cure of the polymer matrix, with the ultimate goal of finding the minimum unit cell size that can be used later for a homogenization procedure to calculate the response of woven fiber textile composites and in particular, fiber tows. For the material properties and measure of randomness under consideration, at least 25 fibers should be used for computations. For smaller numbers of fibers, the standard deviation between different RVEs is larger compared to cases where more then 25 fibers are used. In the context of the proposed curing model, a reduction in randomness measure and a reduction in fiber-volume fraction will lead to a reduction in the relative difference 


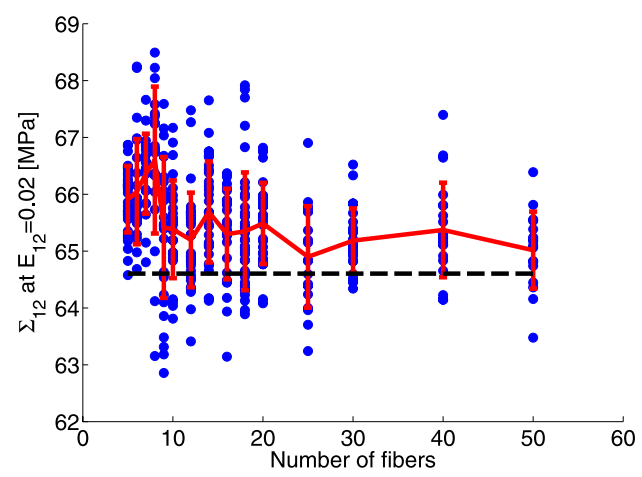

(a) Case $A S: v_{f}=50.0 \%, \bar{\sigma}=10 \%$

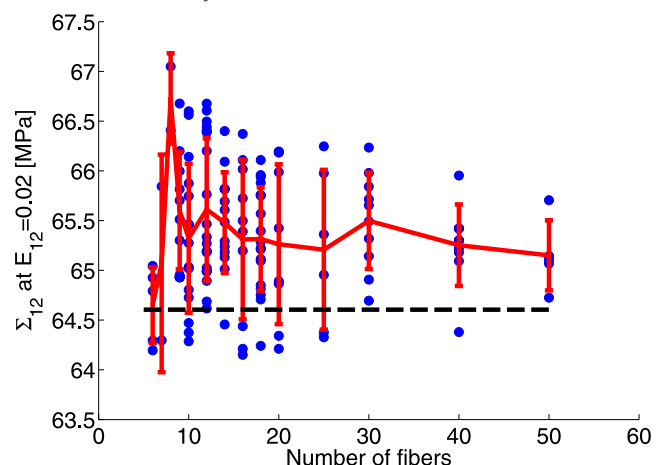

(c) Case $C S: v_{f}=50.0 \%, \bar{\sigma}=15 \%$

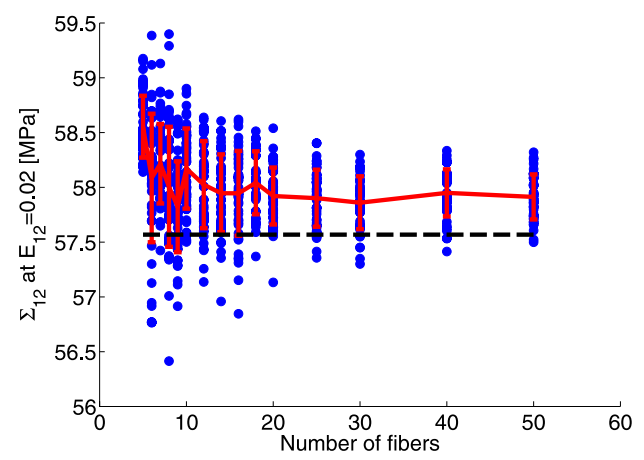

(e) Case ES: $v_{f}=35.0 \%, \sigma=10 \%$

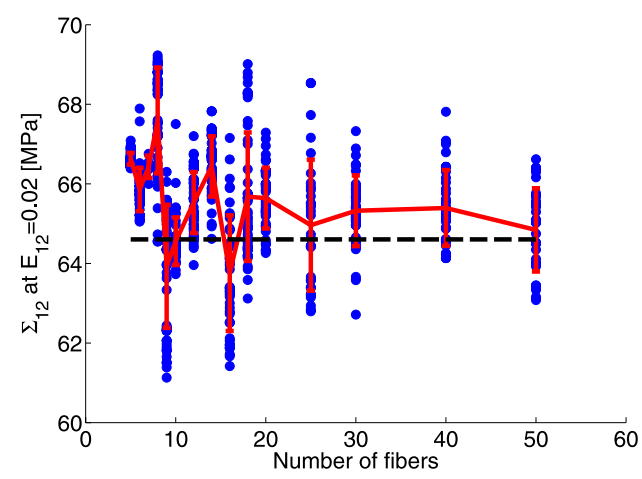

(b) Case $B S: v_{f}=50.0 \%, \bar{\sigma}=5 \%$

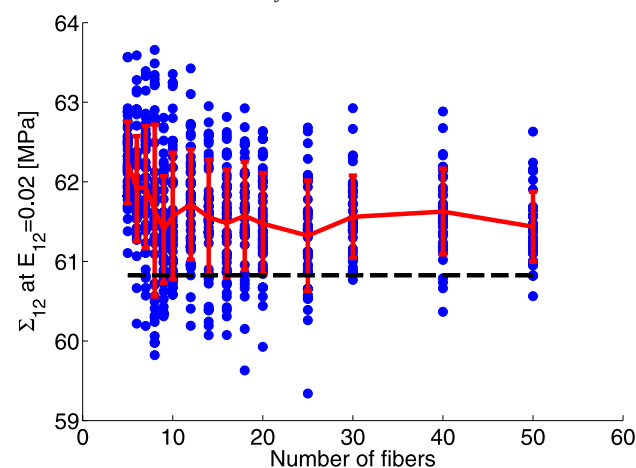

(d) Case $D S: v_{f}=42.5 \%, \bar{\sigma}=10 \%$

- Simulation results
- Average and standard deviation
- - Hexagonal response

Figure 12. Change of the shear stresses versus unit cell size for different volume fractions and degrees of randomness.

between a random packing and hexagonal packing arrangement. The convergence behavior of the curing RVE has been compared to that of an elastic-plastic RVE that is sheared. The convergence behavior is similar, despite the fact that the two BVPs are unrelated. These results have implications for analyses related to damage and failure predictions of polymer matrix fiber composites, since extreme properties dictate the onset of damage and failure, whereas, average values can be used for stiffness predictions. In particular, the need to use RVEs that contain multiple fibers and reflect the statistical aspects of the composite material is recommended for computational schemes that adopt multiscale features. This is of importance in the integrated materials science and engineering (ICME) of polymer matrix composites. In 


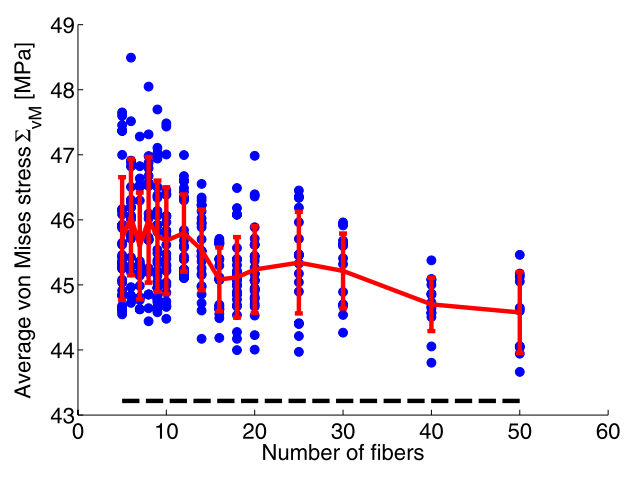

(a) Case $A C: v_{f}=50.0 \%, \bar{\sigma}=10 \%$

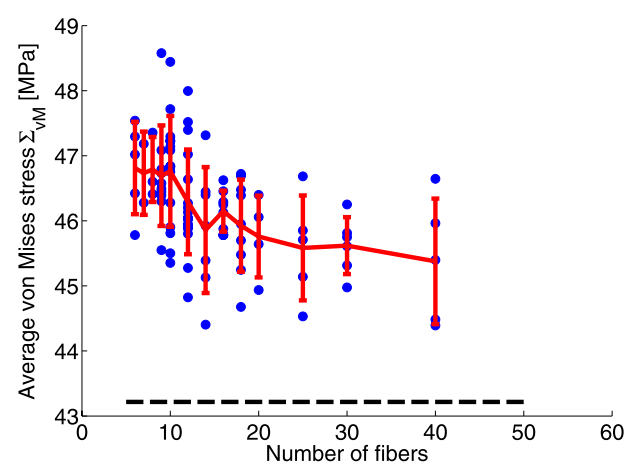

(c) Case $A C: v_{f}=50.0 \%, \sigma=10 \%$

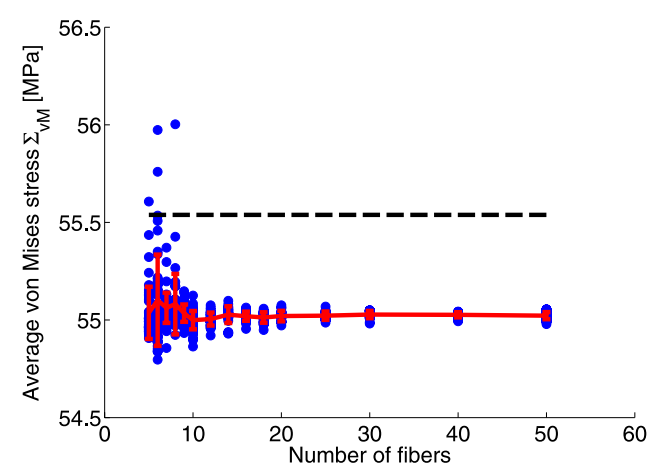

(e) Case AC: $v_{f}=50.0 \%, \sigma=10 \%$

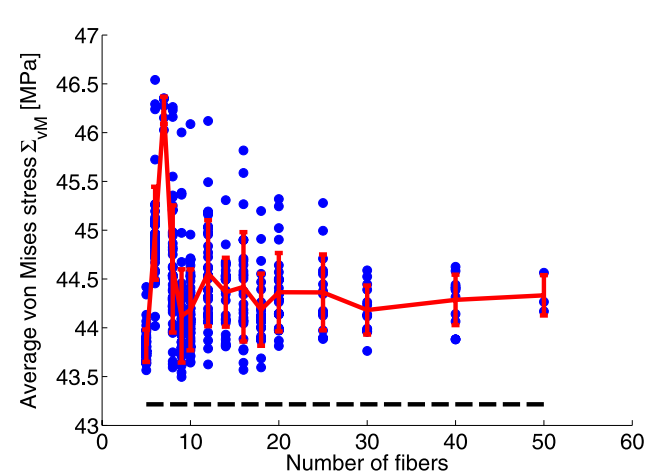

(b) Case $A C: v_{f}=50.0 \%, \bar{\sigma}=10 \%$

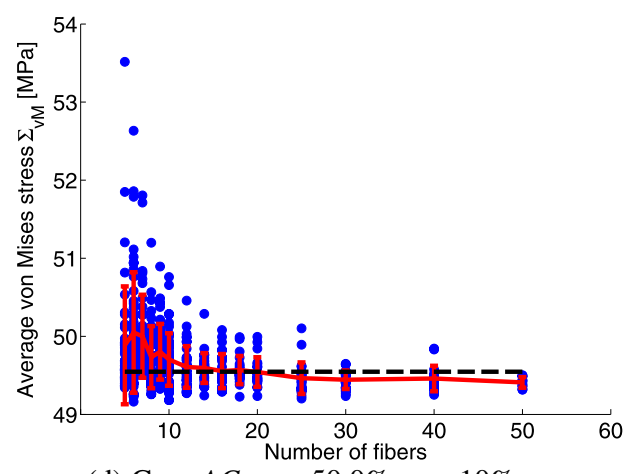

(d) Case $A C$ : $v_{f}=50.0 \%, \sigma=10 \%$

Figure 13. Change of curing stresses vs. unit cell size for different volume fractions and degrees of randomness.

this regard, recent work that use multiple fiber RVEs and the enhanced generalized method of cells [62] for predicting damage and failure in PMCs is noted.

\section{Acknowledgments}

The authors would like to acknowledge the support of the Automotive Composites Consortium (ACC) and its Predictive/Virtual Technology Development and Crash Energy Management 
Group (ACC100). This material is based upon work supported by the Department of Energy under Award Number DE-FC26-02OR22910. The authors are also grateful for the financial support of the Department of Aerospace Engineering, University of Michigan.

\section{Disclaimer}

This report was prepared as an account of work sponsored by an agency of the United States Government. Neither the United States Government nor any agency thereof, nor any of their employees, makes any warranty, express or implied, or assumes any legal liability or responsibility for the accuracy, completeness, or usefulness of any information, apparatus, product, or process disclosed, or represents that its use would not infringe privately owned rights. Reference herein to any specific commercial product, process, or service by trade name, trademark, manufacturer, or otherwise does not necessarily constitute or imply its endorsement, recommendation, or favoring by the United States Government or any agency thereof. The views and opinions of authors expressed herein do not necessarily state or reflect those of the United States Government or any agency thereof.

\section{References}

[1] Lee E H, Rogers T G and Woo T C 1965 Residual stresses in a glass plate cooled symmetrically from both surfaces J. Am. Ceram. Soc. 48 480-7

[2] Rabearison N, Jochum C and Grandidier J 2009 A FEM coupling model for properties prediction during the curing of an epoxy matrix Comput. Mater. Sci. 45 715-24 Proc. 17th Int. Workshop on Computational Mechanics of Materials-IWCMM-17 (Paris)

[3] Corden T J, Jones I A, Jones D T and Middleton V 1998 The mechanisms of interlaminar cracking in thick resin transfer moulded composite cylinders Compos. A: Appl. Sci. Manuf. 29 455-64

[4] Plepys A and Farris R 1990 Evolution of residual stresses in three-dimensionally constrained epoxy resins Polymer 31 1932-6

[5] Darrow D A and Smith L V 2002 Isolating components of processing induced warpage in laminated composites J. Compos. Mater. 36 2407-19

[6] Fernlund G, Rahman N, Courdji R, Bresslauer M, Poursartip A, Willden K and Nelson K 2002 Experimental and numerical study of the effect of cure cycle, tool surface, geometry, and lay-up on the dimensional fidelity of autoclave-processed composite parts Compos A: Appl. Sci. Manuf. 33 341-51

[7] Albert C and Fernlund G 2002 Spring-in and warpage of angled composite laminates Compos. Sci. Technol. 62 1895-912

[8] Johnston A, Vaziri R and Poursartip A 2001 A Plane strain model for process-induced deformation of laminated composite structures J. Compos. Mater. 35 1435-69

[9] Kim J W, Lee J H, Kim H G, Kim H S and Lee D G 2006 Reduction of residual stresses in thick-walled composite cylinders by smart cure cycle with cooling and reheating Compos. Struct. 75 261-6 13th Int. Conf. on Composite Structures-ICCS/13

[10] Kim K and Hahn H 1989 Residual stress development during processing of graphite/epoxy composites Compos. Sci. Technol. 36 121-32

[11] White S and Hahn H 1993 Cure cycle optimization for the reduction of processing-induced residual stresses in composite materials J. Compos. Mater. 27 1352-78

[12] Song S, Waas A M, Shahwan K W, Xiao X and Faruque O 2007 Braided textile composites under compressive loads: modeling the response, strength and degradation Compos. Sci. Technol. 67 3059-70

[13] Brockenbrough J, Suresh S and Wienecke H 1991 Deformation of metal-matrix composites with continuous fibers: geometrical effects of fiber distribution and shape Acta Metall. Mater. 39 735-52

[14] Rossoll A, Moser B and Mortensen A 2005 Longitudinal deformation of fibre reinforced metals: influence of fibre distribution on stiffness and flow stress Mech. Mater. 37 1-17

[15] Herakovich C T 1997 Mechnics of Fibrous Composites (New York: Wiley)

[16] Hill R 1964 Theory of mechanical properties of fibre-strengthened materials: I. elastic behaviour J. Mech. Phys. Solids 12 199-212

[17] Aboudi J 1989 Micromechanical analysis of composites by the method of cells Appl. Mech. Rev. 42 193-221 
[18] Paley M and Aboudi J 1992 Micromechanical analysis of composites by the generalized cells model Mech. Mater. 14 127-39

[19] Bednarcyk B A and Arnold S M 2001 Micromechanics-based deformation and failure prediction for longitudinally reinforced titanium composites Compos. Sci. Technol. 61 705-29

[20] Bednarcyk B A and Arnold S M 2002 Fully coupled micro/macro deformation, damage, and failure prediction for SIC/TI-15-3 laminates J. Aerospace Eng. 15 74-83

[21] Grufman C and Ellyin F 2007 Determining a representative volume element capturing the morphology of fibre reinforced polymer composites Compos. Sci. Technol. 67 766-75

[22] Lissenden C J, Arnold S M and Iyer S K 2000 Flow/damage surfaces for fiber-reinforced metals having different periodic microstructures Int. J. Plast. 16 1049-74

[23] González C and LLorca J 2007 Mechanical behavior of unidirectional fiber-reinforced polymers under transverse compression: Microscopic mechanisms and modeling Compos. Sci. Technol. 67 2795-806

[24] Swaminathan S, Ghosh S and Pagano N J 2006 Statistically equivalent representative volume elements for unidirectional composite microstructures: I. Without damage J. Compos. Mater. 40 583-604

[25] Swaminathan S and Ghosh S 2006 Statistically equivalent representative volume elements for unidirectional composite microstructures: II. With interfacial debonding J. Compos. Mater. 40 605-21

[26] Rakow J F and Waas A M 2004 Size effects in metal foam cores for sandwich structures AIAA J. 427

[27] Bulsara V, Talreja R and Qu J 1999 Damage initiation under transverse loading of unidirectional composites with arbitrarily distributed fibers Compos. Sci. Technol. $59673-82$

[28] Moulinec H and Suquet P 1998 A numerical method for computing the overall response of nonlinear composites with complex microstructure Comput. Methods Appl. Mec. Eng. 157 69-94

[29] Michel J, Moulinec H and Suquet P 1999 Effective properties of composite materials with periodic microstructure: a computational approach Comput. Methods Appl. Mec. Eng. 172 109-43

[30] Buryachenko V A, Pagano N J, Kim R Y and Spowart J E 2003 Quantitative description and numerical simulation of random microstructures of composites and their effective elastic moduli Int. J. Solids Struct. 40 47-72

[31] Ghosh S, Nowak Z and Lee K 1997 Quantitative characterization and modeling of composite microstructures by voronoi cells Acta Mater. 45 2215-34

[32] Heinrich C, Aldridge M, Wineman A S, Kieffer J and Waas A M 2011 Integrated computational materials science and engineering of textile polymer composites 52th AIAA/ASME/ASCE/AHS/ASC Structures, Structural Dynamics, and Materials Conf. (Denver, CO, 2011) AIAA AIAA-2011-1745

[33] Heinrich C, Aldridge M, Wineman A S, Kieffer J, Waas A M and Shahwan K W 2012 Investigation of the response of braided composites during cure and service, in preparation

[34] Ramakrishnan B, Zhu L and Pitchumani R 2000 Curing of composites uning internal resistive heating J. Manuf. Sci. Eng. 122 124-31

[35] Shanku R, Vaughan J and Roux J 1997 Rheological characteristics and cure kinetics of EPON 862/W epoxy used in pultrusion Adv. Polymer Technol. 16 297-311

[36] O'Brien D J and White S R 2003 Cure kinetics, gelation, and glass transition of bisphenol f epoxide Polym. Eng. Sci. 43 863-74

[37] Kamal M R 1974 Thermoset characterization for moldability analysis Polym. Eng. Sci. 14 231-9

[38] Fung Y 1994 A First Course in Continuum Mechanics (Englewood Cliffs, NJ: Prentence Hall) 3rd edn

[39] Doghri I 2000 Mechanics of Deformable Solids (Berlin: Springer)

[40] Ramos J, Pagani N, Riccardi C, Borrajo J, Goyanes S and Mondragon I 2005 Cure kinetics and shrinkage model for epoxy-amine systems Polymer 46 3323-8

[41] Beyer M K and Clausen-Schaumann H 2005 Mechanochemistry:the mechanical activation of covalent bonds Chem. Rev. 105 2921-48

[42] Ikegawa N, Hamada H and Maekawa Z 1996 Effect of compression process on void behavior in structural resin transfer molding Polym. Eng. Sci. 36 953-62

[43] Rudd C 2001 21-Composites Resin transfer modling and strutural reaction injection molding ASM Handbook (ASM International) pp 493-500

[44] Revello M, Saggese L and Gaiero E 2000 Compression molding of SMCs Comprehensive Composite Materials (Amsterdam: Elsevier) pp 763-805

[45] Mai Y, Yee A F, Wineman A S and Xiao C 1998 Stress evolution during thermoset cure Material Research Society Symp. Proc. (San Francisco, CA) p 515

[46] Mei Y 2000 Stress evolution in a conductive adhesive during curing and cooling PhD Thesis University of Michigan, Ann Arbor, MI

[47] Hossain M, Possart G and Steinmann P 2009 A small-strain model to simulate the curing of thermosets Comput. Mech. 43 769-79 
[48] Chike K E, Myrick M L, Lyon R E and Angel S M 1993 Raman and near-infrared studies of an epoxy resin Appl. Spectrosc. 47 1631-5

[49] Merad L, Cochez M, Margueron S, Jauchem F, Ferriol M, Benyoucef B and Bourson P 2009 In-situ monitoring of the curing of epoxy resins by raman spectroscopy Polym. Testing 28 42-5

[50] Yamura H, Matsukawa M, Otani T and Ohtori N 1999 Brillouin scattering study on the elastic properties of epoxy adhesive layer Japan. J. Appl. Phys. 38 3175-8

[51] Sui L, Huang L, Podsiadlo P, Kotov N A and Kieffer J 2010 Brillouin light scattering investigation of the mechanical properties of layer-by-layer assembled cellulose nanocrystal films Macromolecules 43 9541-8

[52] Sanctuary R, Philipp M, Kieffer J, Muller U, Possart W and Kruger J K 2010 Trans-interfacial polymerization and matter transport processes in epoxy-alumina nanocomposites visualized by scanning brillouin microscopy $J$. Phys. Chem. B 114 8396-404 PMID: 20521803

[53] Philipp M, Muller U, Sanctuary R, Kieffer J, Possart W and Kruger J K 2011 On the interplay between matter transport and structure formation at epoxy-hardener interfaces visualized by scanning brillouin microscopy Soft Matter 7 118-24

[54] O'Brian D J, Mather P T and White S R 2001 Viscoelastic properties of an epoxy resin during cure J. Compos. Mater. 35 883-904

[55] Li C, Potter K, Wisnom M R and Stringer G 2004 In-situ measurement of chemical shrinkage of my750 epoxy resin by a novel gravimetric method Compos. Sci. Technol. 64 55-64

[56] Adolf D and Chambers R 1997 Verification of the capability for quantitative stress prediction during epoxy cure Polymer 38 5481-90

[57] Lange J, Toll S, Månson J A E and Hult A 1997 Residual stress build-up in thermoset films cured below their ultimate glass transition temperature Polymer 38 809-15

[58] Simulia 2009 Abaqus 6.9 Manual Dassault Systems

[59] Kulkarni R and Ochoa O 2006 Transverse and longitudinal CTE measurements of carbon fibers and their impact on interfacial residual stresses in composites J. Compos. Mater. $40733-54$

[60] Zaoui A 2002 Continuum micromechanics: Survey J. Eng. Mech. 128 808-16

[61] Song S 2007 Compression response of tri-axially braided textile composites PhD Thesis University of Michigan, Ann Arbor

[62] Pineda E, Bednarcyk B, Waas A and Arnold S 2012 Mesh objective progressive failure of a unidirectional fiber-reinforced composite using the method of cells Int. J. Solids Struct. 